\title{
The Sensory Perception Quotient (SPQ): development and validation of a new sensory questionnaire for adults with and without autism
}

\author{
Teresa Tavassoli ${ }^{1,2^{*}}$, Rosa A Hoekstra ${ }^{1,3}$ and Simon Baron-Cohen ${ }^{1,4}$
}

\begin{abstract}
Background: Questionnaire-based studies suggest atypical sensory perception in over $90 \%$ of individuals with autism spectrum conditions (ASC). Sensory questionnaire-based studies in ASC mainly record parental reports of their child's sensory experience; less is known about sensory reactivity in adults with ASC. Given the DSM-5 criteria for ASC now include sensory reactivity, there is a need for an adult questionnaire investigating basic sensory functioning. We aimed to develop and validate the Sensory Perception Quotient (SPQ), which assesses basic sensory hyper- and hyposensitivity across all five modalities.
\end{abstract}

Methods: A total of 359 adults with $(n=196)$ and without $(n=163)$ ASC were asked to fill in the SPQ, the Sensory Over-Responsivity Inventory (SensOR) and the Autism-Spectrum Quotient (AQ) online.

Results: Adults with ASC reported more sensory hypersensitivity on the SPQ compared to controls $(P<.001)$. SPQ scores were correlated with AQ scores both across groups $(r=.-38)$ and within the ASC $(r=-.18)$ and control groups $(r=-.15)$. Principal component analyses conducted separately in both groups indicated that one factor comprising 35 items consistently assesses sensory hypersensitivity. The SPQ showed high internal consistency for both the total SPQ (Cronbach's alpha $=.92)$ and the reduced 35-item version (alpha $=.93)$. The SPQ was significantly correlated with the SensOR across groups $(r=-.46)$ and within the ASC $(r=-.49)$ and control group $(r=-.21)$.

Conclusions: The SPQ shows good internal consistency and concurrent validity and differentiates between adults with and without ASC. Adults with ASC report more sensitivity to sensory stimuli on the SPQ. Finally, greater sensory sensitivity is associated with more autistic traits. The SPQ provides a new tool to measure individual differences on this dimension.

Keywords: autism spectrum conditions, sensory questionnaire, sensory perception quotient

\section{Background}

In addition to the classic diagnostic criteria (social and communication difficulties alongside unusually narrow interests and repetitive behaviour) [1] atypical sensory reactivity is now also recognized as being at the core of autism spectrum conditions (ASC) [2,3]. Under Symptom $\mathrm{B}$ the new autism criteria for DSM-5 include 'Hyper- or hyporeactivity to sensory input or unusual interests in sensory aspects of the environment (for

\footnotetext{
* Correspondence: teresa.tavassoli@mssm.edu

'Department of Psychiatry, Autism Research Centre, Cambridge University,

18b Trumpington Road, Cambridge CB2 8AH, UK

${ }^{2}$ Seaver Autism Center, Icahn School of Medicine, 1428 Madison Avenue,

10129 New York, USA

Full list of author information is available at the end of the article
}

example, apparent indifference to pain/temperature, adverse response to specific sounds or textures, excessive smelling or touching of objects, visual fascination with lights or movement.' [4]. The study of atypical sensory reactivity in individuals with autism is important given how common this is in ASC. Questionnaires are widely used to study sensory reactivity issues in children with and without ASC [5], in Sensory Processing Disorder [6] and other conditions [7]. Sensory questionnaire studies in ASC mainly record parental reports of their child's sensory experience [5,8-16]. Although parent reports are an important tool, self-report is also crucial, as sensory experiences are by definition subjective. Only a few questionnaire studies have investigated sensory reactivity issues in adults with ASC $[17,18]$. 
The most widely used measure for adults with ASC is the Adolescent/Adult Sensory Profile (AASP), a 60-item self-report measure that finds differences in sensory processing in more than $90 \%$ of adults with ASC $[17,19]$. There have been several studies using the AASP in ASC; one of them found that older individuals with ASC are more similar to control groups than are younger individuals [20]. In addition, adults with schizophrenia and bipolar disorder also show differences on the AASP compared to controls [21]. The AASP is a useful tool because it can be used in clinical settings and describes the particular sensory problems of an individual by assessing specific sensory types, such as sensory sensation seeking.

However, items on the AASP include questions about other factors that may influence our sensory experiences. For example, there are items about visual attention (for example, 'I miss the street, building, or room signs when trying to go somewhere new'), and affective reactions towards sensory stimuli (for example, 'I become frustrated when trying to find something in a crowded drawer'). As such, the AASP, while producing clear group differences, measures a broader set of perceptual processes and affective responses and not basic sensory function. Thus, there is a need for a more fine-grained questionnaire to dissect each perceptual process with greater precision.

More recently the Sensory Over-responsivity Scale (SensOR) was developed to assess sensory processing disorder (SPD), or more narrowly sensory over-responsivity [6]. Sensory Over-Responsivity (SOR) is defined as an exaggerated response to one or more types of sensory stimuli [6]. The SensOR measures SOR by asking how many sensations are experienced as aversive (for example, labels in clothes). The SensOR was developed in combination with an examiner-administered assessment of response to real-world stimuli; these two measures correlate moderately $(r=.47)[6]$. This association suggests that the SensOR is a valid and reliable tool to investigate sensory issues. However, like the Sensory Profile, items on the SensOR include affective reactions towards sensory stimuli (for example, participants have to rate which items in the environment bother them), so again, it is not measuring basic sensory sensitivity.

There is thus a need for a basic sensory perception questionnaire that does not assess social and affective aspects. In contrast to the SensOR and the Adult Sensory Profile, the Sensory Perception Quotient (SPQ), reported here for the first time, only investigates basic sensory sensitivity, with no reference to affective response (see Table 1). The SPQ was developed to quantify individual differences in sensory perception in the general population and adults with ASC, based on the assumption that this trait shows variance following a normal distribution in community samples. All five of the main sensory modalities (vision, hearing, touch, smell and taste) were included.

The purpose behind development of the SPQ is to assist researchers studying sensory perception in adults. Additionally, the SPQ is intended to be useful for occupational therapists and other clinicians. In summary, the objectives of the current study were: (1) to explore the factor structure of the new SPQ, its reliability and its concurrent validity with a previously validated instrument; (2) to investigate if adults with and without ASC show differences on the SPQ; and (3) to explore if sensory sensitivity is correlated with autistic traits, both across and within groups.

\section{Methods}

The Cambridge University Psychology Research Ethics Committee approved the study.

\section{Participants}

Adults with ASC were recruited via an online volunteer database hosted by the Autism Research Centre, University of Cambridge. These volunteers were invited to take part in the online questionnaire study via the website at www.autismresearchcentre.com. Data from participants with no ASC diagnosis were collected via a parallel website at www.cambridgepsychology.com. Only participants who reported to have no psychiatric history were included in the control group.

All participants first filled in background information including age, sex, and history of psychiatric conditions including when, where and by whom they were diagnosed. Only participants with an ASC diagnosis made by a qualified professional (psychologist or psychiatrist) were included in the ASC group. To validate diagnoses in the ASC group and to screen control participants, we

Table 1 Examples of differences between the adult sensory profile (ASP), the sensory over-responsivity scale (SensOR) and the sensory perception quotient (SPQ)

\begin{tabular}{llll}
\hline SPQ Item & ASP item & SensOR item & Difference \\
\hline $\begin{array}{lll}\text { I would be able to tell when an elevator/lift } \\
\text { started moving. }\end{array}$ & $\begin{array}{l}\text { I avoid escalators and/or } \\
\text { elevators because I } \\
\text { dislike the movement. }\end{array}$ & $\begin{array}{l}\text { These aspects related to } \\
\text { movement bother me - }\end{array}$ & $\begin{array}{l}\text { going up or down escalators } \\
\text { perception (for example, detection). }\end{array}$ \\
$\begin{array}{lll}\text { If I look at a pile of blue sweaters in a shop } \\
\text { that are meant to be identical, I would be } \\
\text { able to see differences between them. }\end{array}$ & $\begin{array}{l}\text { that have bright lights } \\
\text { and that are colourful. }\end{array}$ & $\begin{array}{l}\text { These visual sensations bother me: } \\
\text { brightly coloured or patterned materials } \\
\text { (for example, clothes, drapes, wallpaper) }\end{array}$ \\
\hline
\end{tabular}


used the Autism Spectrum Quotient (AQ) [22]. We used a standard inclusion criterion of an AQ cut-off score of 26 and above for the ASC group, and a score below 26 for the control group [22,23]. Nine participants with ASC and 43 control participants were excluded on the basis of the AQ cut-off score, leaving 359 participants: $n$ = 196 participants with ASC (100 males, 96 females) and $\mathrm{n}=163$ control participants (49 males, 114 females). Participants were not reimbursed for taking part in the current study.

\section{Autism Spectrum Quotient}

All participants completed the adult version of the AQ. The AQ is a short, 50-item questionnaire measuring autistic traits, with five subscales (social skills, attention switching, attention to detail, imagination and communication) [22,23]. A score of 0 is assigned to the responses 'definitely agree' and 'slightly agree' and a score of 1 for 'slightly disagree' and 'definitely disagree' for half the items, and the reverse for the other half, designed to avoid a response bias. Total scores could therefore range from 0 to 50, with higher scores indicating more autistic traits. Results from the AQ have been replicated cross culturally $[24,25]$ and across different ages [26]. The AQ shows also good test-retest reliability [22-24].

\section{Raven's Progressive Matrices Test}

IQ was estimated using a short online adaptation of the Raven's Progressive Matrices (60 items) as a timed performance task [27]. The Raven's Progressive Matrices Test has the advantage of being language-free and it can be used for a wide range of ages and cross-culturally (Raven, 2000). Each item consisted of a pattern consisting of a missing section, and the participant is asked to select the option that accurately completes the pattern shown (with 15 seconds allowed for each item).

\section{Sensory over-responsivity scale}

The SensOR Scale consists of a self-report inventory that measures over-responsivity in several sensory domains (touch, vision, hearing, smell, taste, and proprioception) [6]. Participants indicated which items in their daily environment bother them (for example, in the tactile domain: labels in clothing, or in the auditory domain: a clock ticking). The SensOR went through item analysis and reduction prior to item selection for this edition. The current SensOR edition consists of 76 items (28 tactile items, 20 auditory items, 9 taste items, 9 movement items, 5 vision and 5 smell items). The internal consistency reliability for the total test is high $(r=.97)$ and concurrent validity of the SensOR score with the Sensory Profile score for sensory reactivity and sensory avoiding is moderate $(\mathrm{r}=.50)$ [6].

\section{Sensory Perception Quotient (SPQ): Instrument development}

We first generated items for vision, hearing, touch, smell and taste. Next, these items were given to experts in ASC and participants with and without ASC for feedback on wording and applicability. Words with affective aspects (such as like/dislike) were avoided. Instead we focused on basic detection and/or discrimination abilities (for example, 'I would be able to detect if a strawberry was ripe or not by smell alone'). In addition we checked that where possible, items were worded appropriately given that adults with ASC prefer very specific, clear, unambiguous wording (for example, 'I would notice if someone added 5 grains of salt to my water'). Half the items were worded to identify hypersensitive (that is, low thresholds) and half were worded to identify hyposensitive items (that is, high thresholds), to avoid bias (see Table 2 for a complete item breakdown). When scoring, hyposensitive items were reversed, so that a low total SPQ score indicates more reactivity.

The final version of the SPQ covered items for vision, hearing, touch, smell and taste. We aimed to investigate basic sensory processing and therefore included main receptors for each modality and/or the characteristics of the environment relevant to each sense (see Table 2). We developed questions about receptors from different modalities and corresponding environmental stimuli (for example, Pacinian corpuscles are tactile receptors, which are sensitive to vibrations). The only exception was olfaction since there are too many receptors to develop items for each one [28]. Instead, the main functions of olfaction were included. Equal weightings were assigned to vision, hearing and touch, on the basis that none of the senses is more important than others. However we included slightly fewer items for taste and smell since humans do not tend to depend on chemical senses as much as other animals [28]. Humans are microsmatic, having a poor sense of smell, whereas many animals are macrosmatic, having a good sense of smell [28]. Finally, we included a larger number of items in total, so as to be able to reduce these after an item analysis.

Table 2 Categories and subcategories of the sensory perception quotient (SPQ)

\begin{tabular}{lllll}
\hline \multicolumn{5}{c}{ Main categories } \\
\hline $\begin{array}{l}\text { Touch } \\
(20)^{\mathrm{a}}\end{array}$ & Hearing & Vision & Smell & Taste \\
\hline \multicolumn{5}{c}{ Subcategories } \\
\hline Pressure (5) & Amplitude (5) & Acuity (5) & Social (4) & Salty (4) \\
Temperature (5) & Frequency (5) & Brightness (5) & Danger (4) & Sweet (4) \\
Pain (5) & Vestibular (5) & Colour (5) & Food (4) & Sour (4) \\
Vibration (5) & Complexity (5) & Motion (5) & Neutral (4) & Bitter (4) \\
\hline
\end{tabular}

${ }^{\mathrm{a}}$ In brackets is the number of items per category. 


\section{Scoring}

Participants were asked to indicate to what extent they agreed or disagreed with each statement on a Likert scale $(0=$ strongly agree, $1=$ agree, $2=$ disagree, and $3=$ strongly disagree). All item responses were summed [29], with a lower score indicating higher sensory sensitivity. Advantages of this scale are that it avoids uncertain answers, and it can be used for multidimensional constructs.

\section{Procedure}

Participants could complete the Raven's Progressive Matrices, AQ, SensOR and SPQ tasks online in their preferred order, and were allowed to log out between tests.

\section{Results}

\section{Descriptive statistics}

PASW Statistics 18 was used to analyse the data. Tests of normality (Kolmogorov-Smirnov test; KS) showed that that SPQ scores were normally distributed $(P>.20)$. There was no significant difference between the groups on age or IQ $(P>.05)$. However as expected, the ASC group had a higher mean AQ score than the control group $(\mathrm{t}=42.95, P<.0001)$ (see Table 3$)$.

\section{Principal component analysis}

To investigate the underlying factor structure of the SPQ a principal component analysis (PCA) was conducted, using the Varimax rotation method. The PCA was first run for the data from the control participants, and subsequently repeated in the ASC group. Extraction of underlying dimensions was based on inspection of the scree plots, which suggested that the SPQ is composed of two underlying dimensions in both the control group and the ASC group.

Next, factor loadings were visually inspected. For the control group, most items (38 in total) loaded on Factor 1 , whilst only a few items loaded on Factor 2. Items were retained if they showed a high factor loading $(\geq .35)$ on the one factor and a low loading $(\leq .35)$ on the other. We excluded ambiguous items that showed high loadings $\geq$ .35 on both factors [30]. A separate PCA for the ASC group showed similar results. In this group, 43 items

Table 3 Descriptive characteristics of the Autism Spectrum Condition (ASC) and control groups

\begin{tabular}{lccc}
\hline & $\begin{array}{c}\text { ASC } \\
\text { group }\end{array}$ & $\begin{array}{c}\text { Control } \\
\text { group }\end{array}$ & $\begin{array}{c}\text { Group } \\
\text { difference }\end{array}$ \\
\hline $\mathrm{N}$ & 196 & 163 & - \\
Age in years (SD) & $38.7(12.7)$ & $36.8(12.3)$ & No, $P>.05$ \\
AQ score (range from 0 to 50) & $40.4(5.1)$ & $15.4(6.2)$ & Yes, $P<.0001$ \\
Raven score (range from 0 to 60) & $50.3(10.5)$ & $51.5(7.4)$ & No, $P>.05$
\end{tabular}

AQ, Autism Spectrum Quotient. loaded highly on Factor 1. In addition, 35 items that loaded on Factor 1 in the control group also loaded on Factor 1 in the ASC group (see Table 4). For Factor 2, the loadings across both groups were low and inconsistent. These results suggest that one factor, encompassing 35 items, consistently assesses sensory reactivity traits in both adults with ASC and controls.

\section{Item distribution analysis}

In addition, an item distribution analysis was conducted. Items on which more than $70 \%$ of the participants gave the same response were excluded, since items with very little variance are not informative [31]. Four such items were identified: item, 8, 16, 47 and 54. On these items most participants 'strongly agreed' (scoring 0). None of these items loaded on Factor 1.

\section{Item reduction}

In sum, the PCAs suggest that one factor including 35 items consistently assesses traits related to sensory reactivity in both a control group and a clinical group (see Table 4). All these 35 items show considerable response variation. Out of the 35 items loading onto Factor 1, 10 items assessed reactivity to touch, 10 items assessed smell, 6 vision, 5 hearing, and 4 taste. Most of the items (31 out of 35) were hypersensitive items.

\section{Reliability}

For all 92 items, the split-half reliability was high (SpearmanBrown $=.91, \quad P<.0001)$. Additionally, Cronbach's alpha suggested excellent internal consistency for both the full 92 item version of the SPQ $(\alpha=.92)$ and for the reduced 35-item version $(\alpha=.93)$.

\section{Concurrent validity}

To test the concurrent validity of the SPQ the association with the SensOR was examined. High scores on the SensOR and low scores on the SPQ represent more reactivity to stimuli in the environment. The total SPQ and SensOR correlated moderately $(\mathrm{r}=-.50, P<.0001)$ both across groups and within the ASC $(r=-.49, P$ $=.007)$ and control group $(\mathrm{r}=-.23, P=.004)$ (see Figure 1$)$. The concurrent validity was lower for the item reduced version of the SPQ $(r=-.20, P=.0001)$.

\section{Differences between the groups}

A MANOVA with group and sex as fixed factors showed that groups differed significantly on the SPQ total scores $(\mathrm{F}(6,339)=13.44, P<.0001)($ see Table 5$)$. Post hoc tests showed that groups differed significantly on the total SPQ, the item-reduced version, and for all subscales other than smell. Additionally, sex differences were found for the total SPQ (F $(1)=4.71, P<.005)$, and for smell and taste reactivity; females in both groups had 
Table 4 Item loadings for factors 1 (Fac1) and 2 (Fac2) for all 92 items of the Sensory Perception Quotient (SPQ), and item breakdown of the SPQ domains and subdomains (for example, taste-salty)

Factor loadings and item break down

\begin{tabular}{|c|c|c|c|c|}
\hline \multicolumn{4}{|c|}{ Factor loading } & \multirow[t]{3}{*}{ Item domain } \\
\hline \multicolumn{2}{|c|}{$\begin{array}{l}\text { Control } \\
\text { group }\end{array}$} & \multicolumn{2}{|c|}{$\begin{array}{c}\text { ASC } \\
\text { group }\end{array}$} & \\
\hline$\overline{\text { Fac1 }}$ & $\overline{\text { Fac2 }}$ & $\overline{\text { Fac1 }}$ & $\overline{\text { Fac2 }}$ & \\
\hline .34 & -.18 & .38 & -.35 & Taste-salty \\
\hline .60 & .23 & .42 & -.22 & Smell-social \\
\hline .02 & .15 & .35 & .40 & Taste-sweet \\
\hline .04 & -.01 & .03 & .52 & Touch-pain \\
\hline .09 & .36 & .30 & .48 & Vision-motion \\
\hline-.02 & .22 & .26 & .39 & Hearing-complexity \\
\hline .38 & .25 & .55 & -.11 & Smell-food \\
\hline .31 & .33 & .47 & .09 & Taste-sweet \\
\hline .16 & -.28 & .23 & -.12 & Touch-pain \\
\hline-.02 & -.03 & .13 & .39 & Touch-pain \\
\hline .27 & -.19 & .26 & -.16 & Touch-temperature \\
\hline .49 & .04 & .60 & -.23 & Vision-brightness \\
\hline-.12 & .46 & .33 & .42 & Vision-brightness \\
\hline .48 & .13 & .56 & -.08 & Taste-sour \\
\hline .43 & .30 & .58 & .46 & Smell-danger \\
\hline .04 & .19 & .38 & .65 & Touch-vibration \\
\hline-.30 & .19 & -.63 & .02 & Touch-vibration \\
\hline .01 & .41 & .33 & .21 & Hearing-loudness \\
\hline .43 & .05 & .59 & -.20 & Hearing-loudness \\
\hline .34 & .18 & .60 & .10 & Taste-sweet \\
\hline .48 & .17 & .60 & -.11 & Taste-salty \\
\hline-.32 & .60 & -.08 & .42 & Hearing-complexity \\
\hline .34 & -.61 & .48 & -.47 & Vision-colour \\
\hline .25 & -.51 & .03 & -.41 & Hearing-vestibular \\
\hline .40 & .27 & .52 & .41 & Smell-food \\
\hline-.17 & .42 & -.24 & .33 & Hearing-vestibular \\
\hline .22 & .36 & .24 & .57 & Touch-temperature \\
\hline .20 & -.26 & .23 & .06 & Taste-bitter \\
\hline .30 & .35 & .45 & .42 & Hearing-frequency \\
\hline .27 & .14 & .49 & -.05 & Smell-neutral \\
\hline .50 & -.29 & .57 & .21 & Touch-pressure \\
\hline .43 & -.36 & .57 & .44 & Hearing-frequency \\
\hline .52 & -.32 & .61 & -.46 & Vision-motion \\
\hline .22 & .41 & .52 & .38 & Smell-food \\
\hline .46 & .09 & .58 & -.32 & Touch-temperature \\
\hline .36 & .09 & .44 & -.13 & Touch-pain \\
\hline
\end{tabular}


Table 4 Item loadings for factors 1 (Fac1) and 2 (Fac2) for all 92 items of the Sensory Perception Quotient (SPQ), and item breakdown of the SPQ domains and subdomains (for example, taste-salty) (Continued)

$37 \quad$ I would be able to see the individual blades in a rotating fan even if it was at maximum speed.

$38^{\mathrm{a}} \quad$ I would be able to tell the weight difference between two different coin sizes on the palm of my hand, if my eyes were closed.

39 I wouldn't get dizzy on a carousel/merry-go-round, even at high speed.

40 I can't see written words on a page that other people can see.

41 I would be able to distinguish between two oranges purely by their taste.

$42^{\mathrm{a}} \quad$ I couldn't distinguish a familiar person and a stranger by their smell.

$43^{\mathrm{a}}$ I couldn't detect if bread is stale purely by its smell.

44 I can't tell if my clothes are clean or dirty by smell alone.

$45^{\mathrm{a}} \quad$ I would be able to detect the sound of a vacuum cleaner from any room in a two-storey building.

46 I wouldn't notice the difference between even and uneven ground when driving over it sitting in the back seat of a car.

47 I would be able to drink a cup of boiling water straight after it had been poured from the kettle.

48 I couldn't tell two types of green apples apart purely from their colour.

49 I would be able to distinguish between an old and a new book by their smell.

$50 \quad$ I would be able to read a street sign from a distance of 100 feet (30 metres).

51 I can't tell if cars passing me on the street are going at different speeds.

52 I would be able to notice if someone added 5 grains of sugar to my glass of water.

53 I would have difficulty seeing a single leaf clearly even on a tree that is close up.

54 I wouldn't taste if someone added a whole teaspoon of salt to my glass of water.

$55^{\mathrm{a}} \quad$ I would be able to feel the elastic holding up my socks if I stop and thought about it.

56 I can't taste the difference between ripe and non-ripe fruit.

57 I would be able to stand on one foot for fifteen seconds without wobbling.

$58^{\mathrm{a}} \quad$ I would be able to taste the difference between apparently identical pieces of candy.

$59^{\mathrm{a}}$ I notice the weight and pressure of a hat on my head.

$60^{\mathrm{a}}$ I would feel if a single hair touched the back of my hand.

$61^{\mathrm{a}}$ If I was walking along, I would be able to feel a passing truck's vibrations even if my eyes were closed.

$62^{\mathrm{a}} \quad$ I would be able to smell the smallest gas leak from anywhere in the house.

$63^{\mathrm{a}}$ I wouldn't notice if someone changed their perfume, by smell alone.

64 I would be able to tell when an elevator/lift started moving.

65 I can hear dog whistles very easily in the park.

66 I wouldn't taste the difference between different types of lettuce leaves.

67 I couldn't taste if there were two slices of lemon in my glass of water if I was drinking it with my eyes closed.

$68^{\mathrm{a}}$ I can't go out in bright sunlight without sunglasses.

69 I would be able to read small print, such as a serial number on the back of a DVD, at 10 feet (3 metres) away.

70 I get motion sickness easily (for example, car sickness or sea sickness)

$71^{a} \quad$ I would be able to feel a change in the temperature of a cup of coffee after it had sat for 1 minute.

72 I can't hear very low frequency sounds, such as low voices.

$73^{a} \quad$ I would be the first to hear if there was a fly in the room.

\begin{tabular}{|c|c|c|c|c|}
\hline .29 & -.20 & .26 & -.44 & Vision-motion \\
\hline .42 & .03 & .62 & -.25 & Touch-pressure \\
\hline .03 & .07 & -.02 & .24 & Hearing-vestibular \\
\hline-.12 & .37 & -.14 & .63 & Vision-acuity \\
\hline .48 & .22 & .31 & -.11 & Taste-sour \\
\hline .41 & .25 & .42 & .11 & Smell-social \\
\hline .38 & .06 & .58 & .16 & Smell-food \\
\hline .30 & .35 & .43 & .48 & Smell-neutral \\
\hline .44 & .04 & .51 & -.03 & Hearing-frequency \\
\hline .38 & .31 & .46 & .47 & Touch-vibration \\
\hline-.03 & .04 & .16 & .49 & Touch-pain \\
\hline .31 & .26 & .49 & .36 & Vision-colour \\
\hline .25 & .18 & .58 & -.06 & Smell-neutral \\
\hline-.09 & .29 & .22 & -.01 & Vision-acuity \\
\hline .15 & .35 & .25 & .51 & Vision-motion \\
\hline .37 & -.05 & .35 & -.30 & Taste-sweet \\
\hline .21 & .36 & .34 & .56 & Vision-acuitity \\
\hline .20 & .37 & .23 & .56 & Taste-salty \\
\hline .57 & -.11 & .57 & -.11 & Touch-pressure \\
\hline .21 & .41 & .39 & .56 & Taste-sweet \\
\hline .15 & -.44 & .22 & -.06 & Hearing-vestibular \\
\hline .37 & -.27 & .59 & -.27 & Taste-sweet \\
\hline .49 & -.06 & .61 & -.19 & Touch-pressure \\
\hline .59 & -.08 & .64 & -.20 & Touch-pressure \\
\hline .54 & -.00 & .69 & -.07 & Touch-vibration \\
\hline .64 & -.09 & .71 & -.29 & Smell-danger \\
\hline .43 & .19 & .59 & .23 & Smell-social \\
\hline .25 & .07 & .53 & .10 & Hearing-vestibular \\
\hline .32 & -.15 & .50 & -.30 & Hearing-frequency \\
\hline .45 & .27 & .17 & .36 & Taste-bitter \\
\hline .31 & .19 & .48 & .51 & Taste-sour \\
\hline .47 & -.26 & .55 & -.35 & Vision-brightness \\
\hline-.17 & .02 & .04 & -.38 & Vision-acuity \\
\hline .21 & -.01 & -.01 & -.11 & Vision-motion \\
\hline .51 & .07 & .56 & -.12 & Touch-temperature \\
\hline .09 & .52 & .44 & .27 & Hearing-frequency \\
\hline .61 & -.08 & .70 & -.27 & Hearing-loudness \\
\hline
\end{tabular}


Table 4 Item loadings for factors 1 (Fac1) and 2 (Fac2) for all 92 items of the Sensory Perception Quotient (SPQ), and item breakdown of the SPQ domains and subdomains (for example, taste-salty) (Continued)

\begin{tabular}{|c|c|c|c|c|c|c|}
\hline $74^{a}$ & $\begin{array}{l}\text { If I look at a pile of blue sweaters in a shop that are meant to be identical, I would } \\
\text { be able to see differences between them. }\end{array}$ & .51 & -.26 & .68 & -.25 & Vision-colour \\
\hline $75^{\mathrm{a}}$ & I wouldn't detect a new smell in my house instantly before anyone else. & .55 & .26 & .58 & .19 & Smell-neutral \\
\hline 76 & I have perfect pitch: for example, I could repeat a musical tone without any cue. & -.42 & .04 & -.21 & .16 & Hearing-complex \\
\hline 77 & I would be able to bite into a lemon without any problems. & .15 & -.32 & .18 & .25 & Taste-sour \\
\hline 78 & I wouldn't need to wear a coat in the winter, even when it is zero degrees outside. & -.01 & -.03 & -.12 & -.52 & Touch-temperature \\
\hline 79 & $\begin{array}{l}\text { I wouldn't be able to match the colour of a sweater in the shop with the colour of } \\
\text { my trousers at home. }\end{array}$ & .22 & .07 & .14 & .34 & Vision-colour \\
\hline 80 & I wouldn't hear every single note when listening to music. & .39 & -.01 & .33 & .21 & Hearing-frequency \\
\hline $81^{\mathrm{a}}$ & I would be able to smell the difference between most men and women. & .51 & .25 & .59 & -.19 & Smell-social \\
\hline 82 & I choose to wear muted colours. & .14 & -.33 & .41 & -.31 & Vision-brightness \\
\hline 83 & I listen to music at minimum loudness. & .15 & -.22 & .31 & -.27 & Hearing-loudness \\
\hline $84^{a}$ & I would be able to hear each note in a chord even if there were 10 notes. & .38 & -.13 & .43 & -.25 & Hearing-complexity \\
\hline $85^{\mathrm{a}}$ & I close curtains to avoid bright lights. & .46 & -.45 & .49 & -.37 & Vision-acuity \\
\hline 86 & $\begin{array}{l}\text { I wouldn't be able to hear differences in sound if the same instrument played the } \\
\text { same note at different times. }\end{array}$ & .27 & -.12 & .50 & .20 & Hearing-complex \\
\hline $87^{\mathrm{a}}$ & $\begin{array}{l}\text { I would be able to distinguish two brands of coffee by their smell, even with my } \\
\text { eyes closed. }\end{array}$ & .50 & .01 & .66 & -.16 & Smell-food \\
\hline $88^{\mathrm{a}}$ & I can see dust particles in the air in most environments. & .50 & -.37 & .63 & -.42 & Vision-acuity \\
\hline $89^{\mathrm{a}}$ & $\begin{array}{l}\text { I wouldn't be able to taste the difference between two brands of tomato sauce if } \\
\text { they had different concentrations of salt. }\end{array}$ & .43 & .26 & .60 & .35 & Taste-salty \\
\hline $90^{\mathrm{a}}$ & I would be able to smell the smallest amount of burning from anywhere in the house. & .62 & -.10 & .76 & -.20 & Smell-danger \\
\hline $91^{a}$ & If my mobile phone was vibrating in my pocket I would be quick to sense it. & .43 & -.01 & .59 & .12 & Touch-vibration \\
\hline 92 & I find it difficult to see individual stars on a clear night. & .15 & .21 & .43 & .47 & Vision-acuitity \\
\hline
\end{tabular}

Sensory domains included: vision, hearing, touch, smell and taste. Italic items are linked to high thresholds (hyposensitive). Non-italic items are linked to lower thresholds (hypersensitive).

${ }^{a}$ Items included in the short version of the SPQ (those items with factor loadings > .35 in one factor for both groups and a low loading ( $\leq .35$ ) on the other factor).

lower scores on the SPQ (more sensitive) (see Table 5). Gender had however no effect on SPQ-short scores. Results from the SenSOR are reported elsewhere [32].

\section{Correlation between Sensory Perception Quotient and autistic traits}

The total SPQ was correlated with the AQ across groups $(\mathrm{r}=-.38, P=.0001)$ and within the ASC group $(\mathrm{r}=-.18$, $P=.009$ ), and marginally within the control group $(\mathrm{r}=-.15, P=.06)$. A higher score on the AQ corresponds with more autistic traits, while lower scores on the SPQ suggest a lower sensory threshold and thus a higher sensory sensitivity. The reduced SPQ also correlated with the AQ $(\mathrm{r}=-.14, P=.007)$. There was no correlation between SPQ total scores and age $(P=.58)$ or IQ $(P=.95)$.

\section{Discussion}

The current study reports the development and validation of the Sensory Perception Quotient (SPQ), a new sensory questionnaire that provides a quantitative measurement of individual differences in basic sensory perception. The SPQ shows excellent internal consistency and good validity. Adults with ASC reported being more sensitive than control participants to sensory stimuli in vision, hearing, touch and taste, but not smell. Reliable sensory sensitivity measures for adults with ASC are needed since sensory symptoms are now recognized as being at the core of ASC. Past questionnaire-based studies already highlighted the importance of sensory reactivity in ASC, but often used parent reports [5,8-16]. Since it is easier to judge your own sensory experiences the SPQ is an important new self-report questionnaire. This new questionnaire also adds to a battery of new dimensional measures (the Autism Spectrum Quotient $(\mathrm{AQ})$, the Empathy Quotient (EQ) and the Systemizing Quotient (SQ) ) that seek to provide a metric of the spectrum on which ASC lies, and the relationship between ASC and variation in the general population $[22,33,34]$.

With regards to item analysis, principal component analyses suggest that one factor including 35 items consistently assesses traits related to sensory sensitivity, in both the clinical group and the control group. Both the short 35-item version and the total 92-item version of 


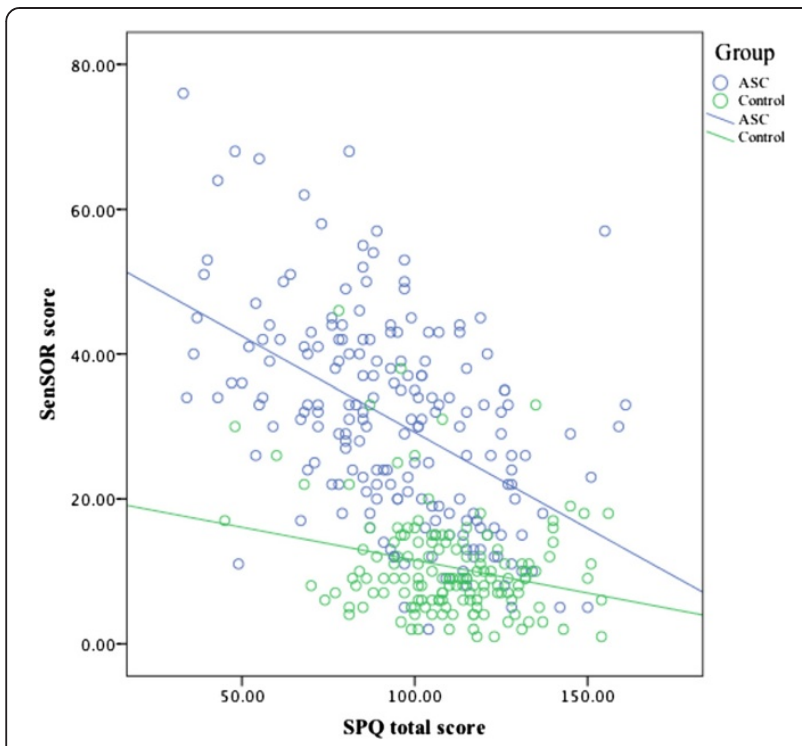

Figure 1 The correlation between the Sensory Perception Quotient (SPQ) with the sensory over-responsivity (SensOR). The lower the score on the SPQ the more sensitive a person is, and the higher the score on the SensOR the more sensitive. ASC, autism spectrum conditions.

the SPQ showed high internal consistency. In addition the SPQ correlated with the AQ, meaning greater sensory reactivity is associated with more autistic traits. Furthermore the SPQ was moderately correlated to a validated sensory scale, the SensOR. It is not surprising that the SPQ and SensOR are not perfectly related, as the SensOR also assesses the affective response to sensory stimuli, whilst the SPQ focuses on basic sensory perception only. Future studies need to test whether SPQ scores correlate to laboratory-measured reactivity measures. In addition, future studies are needed to investigate other test characteristics, such as test-retest reliability.

A limitation of this study is the uneven sex ratio between the groups (more females in the control group). However the ASC and control group differed on the SPQ even when sex was included as a factor. Sex had an effect on the full SPQ, in line with findings from the Sensory Profile [35]. Females in both groups have lower scores on the SPQ, meaning women report being more sensitive. Since females with ASC reported they were more sensitive, the question arises if sensory issues affect females with ASC to a greater extent. A recent study shows that women with ASC report more life-time sensory issues compared to men with ASC [36]. This is important and needs more research since most studies neglect female participants with ASC and mostly include males. Finally, given that this is the first report of the SPQ, future studies are necessary to generate a normative dataset.

\section{Conclusions}

The SPQ is a reliable and valid new tool to measure sensory sensitivity in adults with and without ASC. Adults with ASC report more sensory sensitivity than controls,

Table 5 The mean sensory perception quotient (SPQ) scores before and after item reduction and for all subscales for vision, hearing, touch, smell and taste, for females and males with and without Autism Spectrum Condition (ASC)

\begin{tabular}{|c|c|c|c|c|c|c|c|c|}
\hline & Sex & $\mathrm{SPQ}$ & SPQ & SPQ & SPQ & $\mathrm{SPQ}$ & $\begin{array}{l}\text { SPQ } \\
\text { Smell }\end{array}$ & SPQ \\
\hline & & Full & Short & Vision & Hearing & Touch & & \\
\hline \multirow[t]{6}{*}{ ASC group } & Both & 92.95 & 38.55 & 22.12 & 22.56 & 18.35 & 14.56 & 14.34 \\
\hline & & $( \pm 26.61)$ & (18.68) & $( \pm 6.61)$ & $( \pm 5.41)$ & $( \pm 6.29)$ & $( \pm 8.34)$ & $( \pm 6.39)$ \\
\hline & Male & 97.5 & 40.70 & 22.77 & 23.37 & 23.37 & 15.93 & 15.75 \\
\hline & & $( \pm 25.24)$ & (19.84) & $( \pm 6.36)$ & $( \pm 6.36)$ & $( \pm 5.33)$ & $( \pm 8.29)$ & $( \pm 5.78)$ \\
\hline & Female & 88.21 & 36.25 & 21.54 & 21.54 & 18.58 & 13.12 & 12.87 \\
\hline & & $( \pm 27.30)$ & $( \pm 17.20)$ & $( \pm 6.84)$ & $( \pm 6.84)$ & $( \pm 5.39)$ & $( \pm 8.23)$ & $( \pm 6.69)$ \\
\hline \multirow[t]{6}{*}{ Control group } & Both & 108.96 & 43.01 & 27.12 & 25.85 & 22.74 & 14.85 & 16.25 \\
\hline & & $( \pm 20.53)$ & $( \pm 14.67)$ & $( \pm 5.35)$ & $( \pm 4.79)$ & $( \pm 5.20)$ & $( \pm 5.85)$ & $( \pm 5.09)$ \\
\hline & Male & 110.06 & 43.01 & 27.25 & 25.58 & 22.70 & 16.00 & 17.85 \\
\hline & & $( \pm 17.53)$ & $( \pm 14.67)$ & $( \pm 4.16)$ & $( \pm 4.57)$ & $( \pm 4.46)$ & $( \pm 6.10)$ & $( \pm 5.50)$ \\
\hline & Female & 106.84 & 44.57 & 27.09 & 25.95 & 22.74 & 14.34 & 15.53 \\
\hline & & $( \pm 21.71)$ & $( \pm 14.60)$ & $( \pm 5.82)$ & $( \pm 4.93)$ & $( \pm 5.52)$ & $( \pm 5.69)$ & $( \pm 4.75)$ \\
\hline Group difference? & & $6.71^{\mathrm{a}}$ & $8.20^{\mathrm{a}}$ & $58.33^{a}$ & $26.80^{\mathrm{a}}$ & $41.74^{\mathrm{a}}$ & .89 & $16.80^{\mathrm{a}}$ \\
\hline$F(p)$ & & $(.01)$ & $(004)$ & $(.0001)$ & $(.0001)$ & $(.0001)$ & $(.34)$ & $(.000)$ \\
\hline Sex difference? & & $8.42^{\mathrm{a}}$ & 2.41 & 2.85 & 2.18 & .26 & $6.73^{\mathrm{a}}$ & $21.12^{\mathrm{a}}$ \\
\hline$F(p)$ & & $(.004)$ & $(.12)$ & $(.09)$ & $(.14)$ & $(.60)$ & $(.01)$ & $(.0001)$ \\
\hline
\end{tabular}

Standard deviations are shown in brackets. The SPQ full includes all 92 items and SPQ short includes the 35 item reduced version. In addition, group and sex differences are presented.

${ }^{\text {a }} P$ values $\leq .01$ 
and this may have important implications for how they manage their everyday lives. The SPQ is a useful standardized measure for basic sensory perception in adults with ASC, other clinical disorder and neurotypical adults. Uses of the SPQ include assisting researchers studying sensory issues in adults (for example, in phenotyping studies), and to assist clinicians, such as occupational therapists, in assessing the sensory needs of people with autism.

\section{Abbreviations}

AASP: adolescent/adult sensory profile; ASC: autism spectrum conditions; ASP: adult sensory profile; SenSOR: sensory over-responsivity scale; KS: Kolmogorov-Smirnov test; PCA: principal component analysis; SPQ: sensory perception quotient.

\section{Competing interests}

The authors of this paper report that they have no biomedical financial interests or potential conflicts of interest.

\section{Authors' contributions}

$\Pi$ and SBC designed the SPQ. TT collected the data and $\pi$ and $\mathrm{RH}$ carried out the data analyses. All authors were involved in writing the manuscript and approved the final version.

\section{Acknowledgements}

TT was supported by the Wallace Research Foundation and the Autism Science Foundation. SBC was supported by the MRC UK, the Wellcome Trust, and the Autism Research Trust. This work was conducted in association with the NIHR CLAHRC EoE. We are grateful to the participants for their generous cooperation, and to Dr. Carrie Allison for her help.

\section{Author details}

'Department of Psychiatry, Autism Research Centre, Cambridge University, 18b Trumpington Road, Cambridge CB2 8AH, UK. ${ }^{2}$ Seaver Autism Center, Icahn School of Medicine, 1428 Madison Avenue, 10129 New York, USA. ${ }^{3}$ Department of Life Health and Chemical Sciences, The Open University, Milton Keynes MK7 6AA, UK. ${ }^{4}$ Cambridgeshire and Peterborough NHS Foundation Trust, CLASS Clinic, Cambridge CB21 5EF, UK.

Received: 18 December 2013 Accepted: 31 March 2014 Published: 24 April 2014

\section{References}

1. APA: Diagnostic and Statistical Manual of Mental Disorders. 3rd edition. Washington DC: American Psychiatric Association; 1987.

2. Grandin T: My experiences with visual thinking, sensory problems and communication difficulties. Center Study Autism 1996, Vintage Books.

3. Chamak B, Bonniau B, Jaunay E, Cohen D: What can we learn about autism from autistic persons? Psychother Psychosom 2008, 77:271-279.

4. American Psychiatric Association: Diagnostic and Statistical Manual of Mental Disorders (DSM). 5th edition. 2013.

5. Tomchek SD, Dunn W: Sensory processing in children with and without autism: a comparative study using the short sensory profile. Am J Occup Ther 2007, 61:190-200.

6. Schoen SA, Miller $\sqcup$, Green KE: Pilot study of the Sensory OverResponsivity Scales: assessment and inventory. Am J Occup Ther 2008, 62:393-406.

7. Baranek GT, David FJ, Poe MD, Stone WL, Watson LR: Sensory Experiences Questionnaire: discriminating sensory features in young children with autism, developmental delays, and typical development. J Child Psychol Psychiatry 2006, 47:591-601.

8. Baranek GT, Boyd BA, Poe MD, David FJ, Watson LR: Hyperresponsive sensory patterns in young children with autism, developmental delay, and typical development. AJMR 2007, 112:233-245.

9. Hilton CL, Harper JD, Kueker RH, Lang AR, Abbacchi AM, Todorov A, LaVesser PD: Sensory responsiveness as a predictor of social severity in children with high functioning autism spectrum disorders. $J$ Autism Dev Disord 2010, 40:937-945.
10. Wiggins LD, Robins DL, Bakeman R, Adamson LB: Brief report: sensory abnormalities as distinguishing symptoms of autism spectrum disorders in young children. J Autism Dev Disord 2009, 39:1087-1091.

11. Cheung PP, Siu AM: A comparison of patterns of sensory processing in children with and without developmental disabilities. Res Dev Disabil 2009, 30:1468-1480.

12. Kientz MA, Dunn W: A comparison of the performance of children with and without autism on the Sensory Profile. Am J Occup Ther 1997, 51:530-537.

13. Lane AE, Dennis SJ, Geraghty ME: Brief report: further evidence of sensory subtypes in autism. J Autism Dev Disord 2011, 41:826-831.

14. Ermer J, Dunn W: The sensory profile: a discriminant analysis of children with and without disabilities. Am J Occup Ther 1998, 52:283-290.

15. Lane AE, Young RL, Baker AE, Angley MT: Sensory processing subtypes in autism: association with adaptive behavior. J Autism Dev Disord 2010, 40:112-122.

16. Schoen SA, Miller $L$, Brett-Green BA, Nielsen DM: Physiological and behavioral differences in sensory processing: a comparison of children with autism spectrum disorder and sensory modulation disorder. Front Integr Neurosci 2009, 3:29.

17. Crane L, Goddard L, Pring L: Sensory processing in adults with autism spectrum disorders. Autism 2009, 13:215-228.

18. Kern JK, Trivedi MH, Grannemann BD, Garver CR, Johnson DG, Andrews AA, Savla JS, Mehta JA, Schroeder JL: Sensory correlations in autism. Autism 2007, 11:123-134.

19. Kern G, Grannemann T, Carmody A: Examining sensory quadrants in autism. Res Autism Spectr Disord 2007, 1:185-193.

20. Kern JK, Trivedi MH, Garver CR, Grannemann BD, Andrews AA, Savla JS, Johnson DG, Mehta JA, Schroeder JL: The pattern of sensory processing abnormalities in autism. Autism 2006, 10:480-494.

21. Brown C, Tollefson N, Dunn W, Cromwell R, Filion D: The Adult Sensory Profile: measuring patterns of sensory processing. Am J Occup Ther 2001, 55:75-82.

22. Baron-Cohen S, Wheelwright S, Skinner R, Martin J, Clubley E: The Autism Spectrum Quotient $(\mathrm{AQ})$ : evidence from Asperger syndrome/high functioning autism, males and females, scientists and mathematicians. J Autism Dev Disord 2001, 31:5-17.

23. Baron-Cohen S, Hoekstra RA, Knickmeyer R, Wheelwright S: The AutismSpectrum Quotient (AQ)-Adolescent Version. J Autism Dev Disord 2006 36:343-350.

24. Hoekstra RA, Bartels M, Cath DC, Boomsma DI: Factor structure, reliability and criterion validity of the Autism-Spectrum Quotient (AQ): a study in Dutch population and patient groups. J Autism Dev Disord 2008, 38:1555-1566.

25. Wakabayashi A, Baron-Cohen S, Wheelwright S, Tojo Y: The AutismSpectrum Quotient (AQ) in Japan: A cross-cultural comparison. J Autism Dev Disord 2006, 36:263-270.

26. Auyeung B, Baron-Cohen S, Wheelwright S, Allison C: The Autism Spectrum Quotient: Children's Version (AQ-Child). J Autism Dev Disord 2008, 38:1230-1240

27. Raven J: The Raven's progressive matrices: change and stability over culture and time. Cogn Psychol 2000, 41:1-48.

28. Goldstein AG: Sensation and Perception. Belmont: Publisher Wadsworth; 2002.

29. Hoyle RH, Haris MJ, Judd CM: Research Methods in Social Relations. Belmont, CA: Thomson/Wadsworth; 2002.

30. Lee S, Ma Y, Tsang A: Psychometric properties of the Chinese 15-item Patient Health Questionnaire in the general population of Hong Kong. J Psychosom Res 2011, 71:69-73.

31. Rust J, Golomok S: Modern Psychometrics: The Science of Psychological Assessment., Second edition. London: Routledge; 1999.

32. Tavassoli T, Miller L, Schoen S, Nilson D, Baron Cohen S: Sensory overresponsivity in adults with autism spectrum disoder. Autism 2013. doi:10.1177/ 1362361313477246.

33. Baron Cohen S, Wheelwright S: The Empathy Quotient (EQ). An investigation of adults with Asperger Syndrome or high functioning autism, and normal sex differences. J Autism Dev Disord 2004, 34:163-175.

34. Wakabayashi A, Baron-Cohen S, Wheelwright S, Goldenfeld N, Delaney J, Fine D, Smith R, Weil L: Development of short forms of the Empathy Quotient (EQ-short) and the Systemizing Quotient (SQ-short). Personal Individ Differ 2006, 41:929-940. 
35. Dunn $W$, Westman $K$ : The sensory profile: the performance of a national sample of children without disabilities. Am J Occup Ther 1997, 51:25-34.

36. Lai M, Lombardo M, Pasco G, Ruigrok A, Wheelwright S, Sadek S,

Chakrabarti B, Consortium MRCAIMS, Baron-Cohen S: A behavioral comparison of male and female adults with high functioning autism spectrum conditions. PLoS One 2011, 6:e20835.

doi:10.1186/2040-2392-5-29

Cite this article as: Tavassoli et al:: The Sensory Perception Quotient (SPQ): development and validation of a new sensory questionnaire for adults with and without autism. Molecular Autism 2014 5:29.

\section{Submit your next manuscript to BioMed Central and take full advantage of:}

- Convenient online submission

- Thorough peer review

- No space constraints or color figure charges

- Immediate publication on acceptance

- Inclusion in PubMed, CAS, Scopus and Google Scholar

- Research which is freely available for redistribution 